\title{
Controlling Fic proteins
}

Fic proteins have recently emerged as an important class of effector that bacterial pathogens can use to interfere with host cell signalling pathways. Most of the bacterial Fic proteins characterized so far modify host RHO family GTPases by adenylylation. Writing in Nature, Philipp Engel, Arnaud Goepfert and colleagues now reveal the mechanism by which this post-translational modification is controlled.

Adenylylation of host RHO GTPases interferes with host cell cytoskeletal dynamics, leading to cell death. Previous structural analysis of the effector domain of the Histophilus somni Fic protein, IbpA, had provided researchers with a detailed understanding of the adenylylation reaction and the involvement of the eponymous FIC domain, but little was known about how this reaction was regulated. Engel et al. began by analysing the Bartonella schoenbuchensis Fic protein VbhT, which is encoded within a type IV secretion-associated locus downstream of a short open reading frame, $v b h A$. Heterologous expression of $v b h T$ in Escherichia coli led to filamentation, but co-expression with $v b h A$ led to normal growth, and no VbhT-dependent adenylylation was seen in the presence of VbhA. These results are consistent with VbhT and VbhA being part of a toxin-antitoxin module. Alignment of the VbhA coding sequence with the upstream regions of $>150$ different bacterial Fic genes revealed that this arrangement could be widespread, and a conserved inhibitory motif was identified: (S/T)XXXE(G/N).

The crystal structure of VbhA in complex with the FIC domain of VbhT was solved to a $1.5 \AA$ resolution. VbhA folds into three antiparallel helices, with the aminoterminal helix (designated $\alpha_{\text {inh }}$ ) that

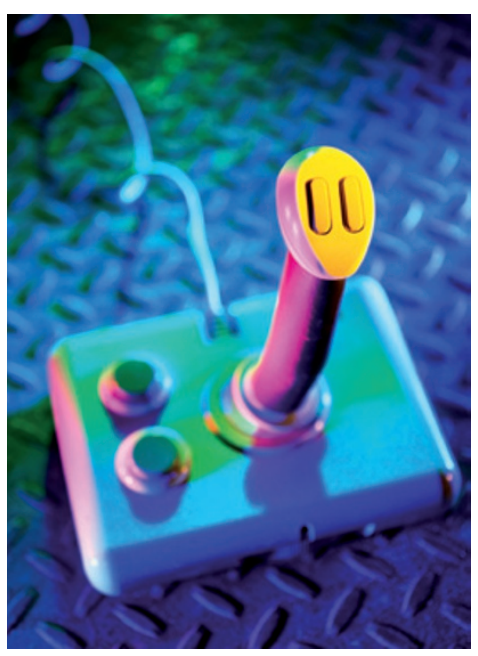

contains the inhibitory motif located close to the VbhT ATP-binding site. Analysis of other bacterial Fic proteins revealed that the structural equivalent of $\alpha_{\text {inh }}$ can actually be part of the FIC domain. This led the authors to specify a tripartite classification system for Fic proteins: class I, in which $\alpha_{\text {inh }}$ is contributed by an antitoxin; class II, in which $\alpha_{\text {inh }}$ is present in the FIC domain as an amino-terminal helix; and class III, in which $\alpha_{\text {inh }}$ is present in the FIC domain as a carboxy-terminal helix. Finally, detailed analysis of the structure and function of $\alpha_{\text {inh }}$ allowed the authors to propose a general mechanism for inhibition of FIC domain adenylylation activity, in which an inhibitory glutamate finger within $\alpha_{\text {inh }}$ obstructs the ATP-binding site.

Structural homology modelling indicates that this mechanism of inhibition is conserved in all three domains of life. Future work will look at how the interaction between $\alpha_{\text {inh }}$ and the FIC domain is weakened in order to activate adenylylation.

Sheilagh Molloy

ORIGINAL RESEARCH PAPER Engel, P. et al. Adenylylation control by intra- or intermolecular active-site obstruction in Fic proteins. Nature $\mathbf{4 8 2}$ 107-110 (2012) 